\title{
A Data Replica Selection Algorithm Based on Cloud Platform
}

\author{
Shaomin Zhang ${ }^{1, a}$, Qi Wang ${ }^{1, b}$, Baoyi Wang ${ }^{1, c}$ \\ ${ }^{1}$ School of Control and Computer Engineering, North China Electric Power University, \\ Baoding,071003, China \\ aemail: zhangshaomin@126.com, bemail: 8785724@qq.com, cemail: wangbaoyiqj@126.com
}

\section{Keywords: Smart Grid Task; MapReduce; Data Replicas; Join Operation}

\begin{abstract}
Aiming at the problem that how to choose the least cost data resources from multiple datasets which have the same contents but different locations for smart grid tasks, a least cost of selection algorithm based on locality is proposed. First, the problem of choosing data replicas is abstract as a shortest path issue that is from the source point to other vertices in directed graph. Second, in order to complete the join operation in Map stage, the related data is grouped in the same datanode, so as to avoid pulling data in Reduce stage. Experimental results show that the algorithm can effectively reduce the data transmission time, and then reduce the task completion time, improve the real-time performance of the smart grid processing tasks.
\end{abstract}

\section{Introduction}

In recent years, with the rapid development of smart grid, the scale of it is more and more large, and the amount of collected data is also increasing. The requirement of security and reliability in data storage is more and more high. For a provincial Power Grid Corp, for 10000 sets of terminal, each collects data once every five minutes, produces about a total of $430 \mathrm{G}$ data per day, the annual production of data is close to $150 \mathrm{~TB}^{[1]}$. With the development of smart grid, these data will continue to increase. Storage system not only safely and reliably to storage these data, but also quickly to deal with these data ${ }^{[2]}$, in order to obtain favorable information. The traditional relational database has been unable to adapt to storage this kind of data because of its poor extension and high cost.

Smart grid data has correlations ${ }^{[1]}$, such as temporal correlation, spatial correlation. For state testing equipment, the source of data participated in the assessment is wide, not only including structured data, but also unstructured audio and video data. In the analysis, it is often necessary to carry on the correlation analysis to a variety of data, it also need to analyze the situation of weather at the same time. The problem that how to query these related data efficiently is need to be solved urgently.

Hadoop ${ }^{[3-5]}$ is an open source distributed computing platform under the flag of the Apache Software Foundation. Hadoop takes the HDFS ${ }^{[4,6]}$ and MapReduce ${ }^{[4,7]}$ as the core, it takes transparent distributed architecture for smart grid data storage. The cloud storage can store data safely and reliably.

At present, many domestic and foreign literatures have done a research on reducing the processing time of the cloud computing. Chinese Academy of Sciences and the Tencent Inc done together in literature[8], they propose a join query processing algorithm $\mathrm{CHMJ}^{*}$, The algorithm uses the consistency hash algorithm for data mapping, for the tables which have connection relationship, it stores data to the same physical node according to the join property, completes the join operation of the data tables locality, which can reduce the data transmission quantity of intermediate results, and improve the efficiency of data query. Srikumar Venugopal and Rajkumar Buyya of Melbourne University in Australia in literature[9] abstracted the selection of data replicas as a set covering problem(SCP) to reduce the amount of remote data transmission, thus reducing the transfer time of data replicas, it can be solved by using tree search algorithm. The literature[10] proposes a data grid copy selection strategy based on the ant algorithm, the algorithm is very good to adapt to the dynamic and the complexity of distributed storage system for it is collaborative and positive feedback. It belongs to a kind of heuristic algorithm, which needs to use the dynamic interaction with the environment to get feedback information to adjust itself, obtaining the optimal 
copy needs a adjustment process. The literature[11] proposes that facing the problem how to choose data resources from the same contents but different locations as input of the application, by building a cloud environment and data selection model, abstracts the cost minimization problem as a weighted set covering problem, this method makes the task completion time and economic cost balance. But solving the problem is a NP-hard problem, it was a little uncertain that makes the optimal solution has limitations, which maybe not a really optimal solution.

Aiming at how to reduce the time of data transmission in the task of smart grid, in this paper, we studied the data replicas selection strategy based on Hadoop cloud platform for smart grid processing tasks. Considering the distribution of the data center of the smart grid, and the MapReduce parallel programming model, we take optimization on cloud storage data selection strategy. On this basis, the related data is stored in the same physical node, according to the locations of data replicas, combining with the knowledge of graph theory, we propose a least cost of selection algorithm based on local data(LCSL). This scheme can make full use of the computing resources and network bandwidth of the cloud platform, and realize the efficient and real-time processing of the smart grid data. Combining with the CloudSim cloud simulator, the experimental test was carried out by constructing the network topology graph, the results prove that this scheme can effectively reduce the time of data processing in smart grid, improve the real-time performance.

\section{Related Work}

\section{Hadoop Data Selection Strategy and Shortages}

The default data selection strategy in Hadoop is based on the distance between the data nodes and the client, according to the number of routing hops, selects the nearest data nodes to return to the client. But when faced with a large data center, and even needed to read data across multiple data centers, it dose not the least hops , the least data transmission time, and if when the transmission link is error in the process of data transmission, it needs to recalculate the shortest distance.

\section{MapReduce Task Process and Shortages}

The MapReduce programming model can be used to develop a distributed computing program which is convenient for the user and without caring about the underlying details. The process is showed in Figure 1, it uses the (key, value) form of key-value pairs as input and output. The Map task makes the data (key, value) that users input to change to a intermediate key-value pair set through the custom mapping process. The intermediate results of Map tasks then are transferred to Reduce task by key-value, then reduce the temporary intermediate key/value pairs, and output the final results.

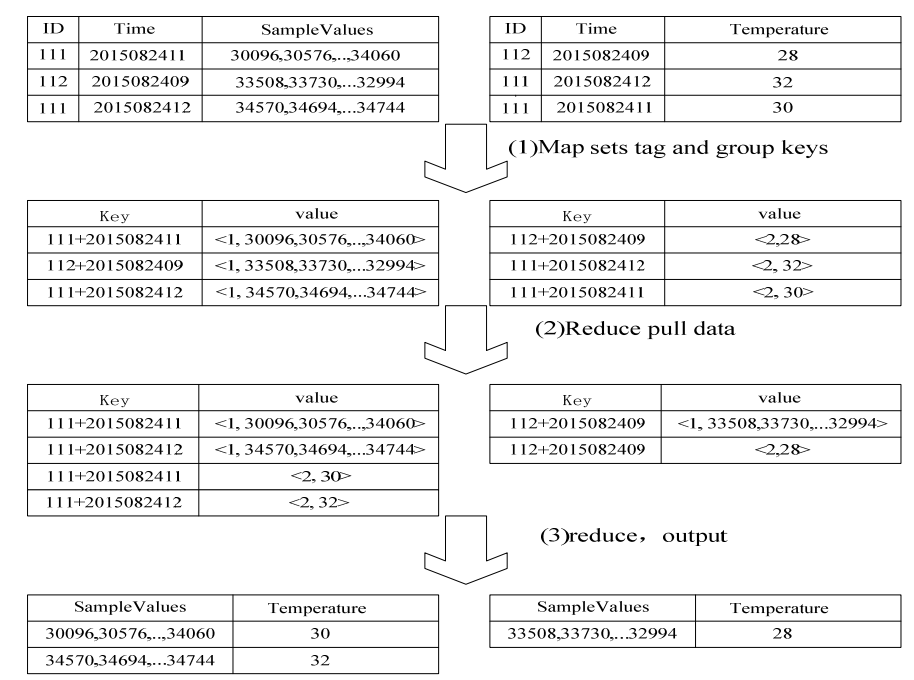

Fig.1. MapReduce process

The model has shortages ,it is showed in Figure 1, for the traditional Hadoop data distribution, the Map phase will produce a great deal of data which has nothing to do with the final join operation, when the related data sets are not in the same node, Reduce tasks in the join operation 
need to pull a large number of irrelevant data, this will add unnecessary data transmission, and also need for multiple grouping sort, that increase the processing time.

Through summarizing the above analysis, it still has challenges for smart grid data processing tasks in data selection strategy. The cloud storage environment based on the Hadoop due to its high reliability and copy technology is suitable for smart grid data storage. According to the characteristics of the smart grid data, and the distribution of the data centers, Hadoop has a large optimization space for smart grid in data storage.

\section{Design of The Local Least Cost Data Selection Algorithm}

Since the MapReduce process and data selection strategy have shortages in Hadoop, it will take unnecessary data transmission in the process of reading data, and choose the maximum cost data replicas for input. This article moves the MapReduce tasks to datanode, and makes join operations in Map stage. According to dijkstra algorithm, it selects the local minimum cost data replicas, as the data input for processing tasks.

\section{Optimization of MapReduce Programming Model}

The traditional parallel programming model MapReduce will produce a large number of intermediate results which have noting to do with the final calculation, that increases the processing task's execution time. In order to reduce the data transmission time in the Reduce process that it pulls large number of data, the model makes the join operations in Map stage, does the join operations in each partition, then gets the final results.

\section{Dijkstra Algorithm}

Dijkstra algorithm is to solve the problem of the shortest path in the directed graph, it can calculate the shortest path from a vertex to the rest of the vertices. For the directed graph $G=(V, E)$, the weight function $W(e)$ for the edge in $\mathrm{G}$, The source point is $V 0$, determines the shortest paths from $V 0$ to other vertices. The algorithm is described as follows:

(1) Structuring adjacency matrix based on the directed graph $\cos t[i][j]=\left\{\begin{array}{c}\langle i, j\rangle \\ \infty\end{array}\right.$

$\langle i, j\rangle$ expresses weights on the arc, $\infty$ expresses there is no arc between $i$ and $j$. Initial $\cos t[i][j]=0$, set $\mathrm{s}$ as a collection that includes the shortest paths from $V 0$ to the rest of vertices, its initial state is empty. At this point, the initial state of the shortest paths from $V 0$ to other vertices $W i$ is that:

$$
\operatorname{dist}[w]=\cos t\left[V_{0}\right][w] w \in V(G)
$$

(2) Selecting $u$ makes $\operatorname{dist}[w]=\min \{\operatorname{dist}[w] \mid w \notin s, w \in V(G)\}$ ( $u$ is a end point of a shortest path that is from $V 0$ ), let $s=s \bigcup\{u\}$ (that is making $u$ into $s$ ).

(3) Modify all the shortest path length whose end are not in s. If (the New choosing shortest path length)

$$
\operatorname{dist}[u]+\cos t[u][w]<\operatorname{dist}[w]
$$

( the shortest path length of other end) then modify $\operatorname{dist}[w]$ as $\operatorname{dist}[w]=\operatorname{dist}[u]+\cos t[u][w]$

(4) Repeat operations (2)、(3) for n-1 times. Thus obtain the shortest paths from $V 0$ to each vertex in $G$.

\section{Design of The Local Least Cost Data Selection Algorithm}

The smart grid data analysis task $\mathrm{J}$, data collection that $\mathrm{J}$ needs is $\mathrm{D}$, the data collection just contains logical file name, each logical file name corresponds to some physical file names. So when $\mathrm{J}$ chooses copies, there will be many different combinations, and different copies, different cost. The cost of each analysis tasks gets copies is $W_{i}$, expressing the time each task gets the data. 
Figure 2 describes the issue that smart grid tasks choose data sets. Task $\mathrm{J}$ need data sets A, B, $\mathrm{C}$, construct a weighted directed graph according to the physical file names of data sets, and the cost of accessing to them. Because the dijkstra algorithm is to solve the shortest path problem in a directed graph, according to the need of the algorithm, this paper constitutes the visit path as a directed graph, makes the access cost as the weights of the directed graph. Abstract model shows in figure 2 .

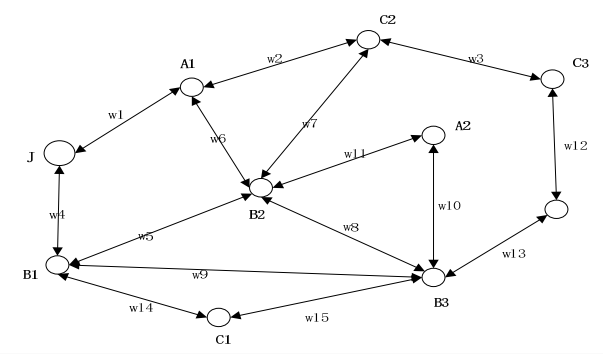

Fig.2. Network topology model for data sets

Above description: because it is a directed graph, for example, vertex A1, B2,A1 is the tail, B2 is the head, another is opposite, in order to draw it simply, we uses two-way arrow to represent this relationship.

Through constructing the abstract model for homologous data sets in different data centers and different frames, we transform the least cost selection strategy to solve $\mathrm{J}$ to each physical file name's shortest distance. And then use dijkstra algorithm to solve it.

\section{Steps of LCSL Algorithm}

Step one: According to the data sets required by analysis task $\mathrm{J}$, gets the physical file name from logical file name in copy management module

Step two: According to the physical file name, abstracted the processing tasks, datanodes which the copies are in them, and the access cost as a weighted directed graph

Step three: According to dijkstra algorithm find the shortest path from $\mathrm{J}$ to each datanode

Step four: According to the shortest path set select the minimum cost data replicas set

Step five: Move Map tasks to data sides, and join the intermediate results

Step six: Return the results to client

\section{Algorithm Performance Analysis}

Cloud computing task completion time is divided into the data processing time $T_{p}$ and data transmission time $T_{t}$, in the same parallel environment, $T_{p}$ is the same, the little $T_{t}$ is, the shorter the task completion time is.

$$
T_{t}=\frac{\text { size of data sets }}{\text { network bandwidth }}
$$

LCSL algorithm, when chose data sets, it gets the least cost data set first, that is network bandwidth is large, and when processing data, it completes join operation in Map side to avoid the unnecessary data transmission, that makes the data sets smaller, so reduces the transmission time, eventually makes the task completion time shorter.

\section{Test results}

\section{Building of Experiment Environment}

In this paper studies the cloud simulator CloudSim, on the basis simulation experiment is carried out. There are four data centers with five frames in each data center in experiments, and link them together with different network bandwidth. Before executing the task, according the Uniform distribution that stored data in different frames in the four data centers, it is uniform distribution, data sets distributed on the data centers in the same probability. Simulation environment copies data according to Hadoop default number for data replicas, each data block has three copies. According to each network bandwidth to define weights in the network topology. 


\section{Analysis of Test Results}

Task completion time

Comparing LCSL algorithm with SCP which is mentioned in literature [9], this paper uses task completion time to test the performance of LCSL algorithm.

Selects different number of data sets, the data sets increases from 1 to 8 , compares the task completion time for LCSL and SCP, the results are showed in Figure 3.

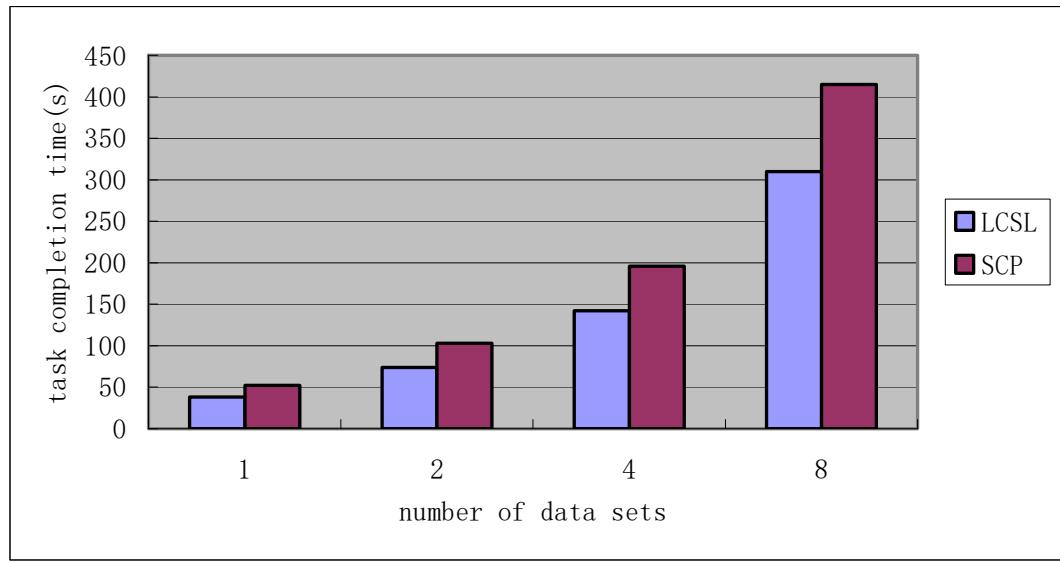

Fig.3. Task completion time comparison

As shown in Figure 3, analyze the task completion time, the time of LCSL is shorter than SCP all the time, specifically, the time is reduced by $27 \%$. It is because that SCP is solved by set covering method, it belonged to a NP problem, the results is not stability. It is doing the best to choose the local data sets, but LCSL chose the least cost data sets, and moves the Map tasks to datanodes, completes the join operation in Map stage, reduces the unnecessary data transmission, thus reduces the data processing time.

Local data analysis of LCSL

Using data transmission time to test the locality of LCSL, it is smaller that shows algorithm can better choose the local data sets as a data input ${ }^{[11]}$.

$$
\text { transmissiontime ratio }=\frac{\text { datatransmissiontime }}{\text { taskcompletiontime }}
$$

Considering the data transmission time ratio, variable is still the number of data sets from 1 to 8, contrasts LCSL and SCP in the transmission time ratio as shown in Figure 4 below.

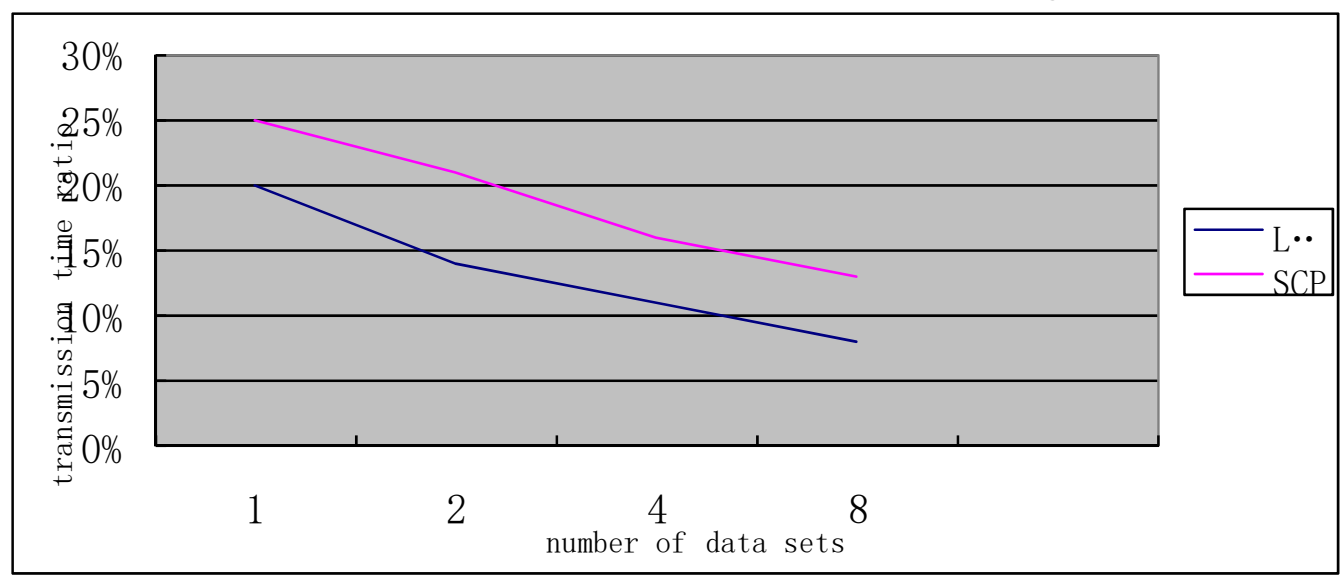

Fig.4. Data transmission ratio

As shown in Figure 4, The data transmission ratio of LCSL is always smaller than SCP, that is because when LCSL chooses data sets, it selects the local data, and moves Map tasks to datanode, does the join operations in Map stage, reduces a lot of unnecessary intermediate results to transport, so reduces the data transmission time. 


\section{Conclusion}

In this paper, we study the MapReduce parallel programming model and data selection strategy in Hadoop. Data selection algorithm LCSL is proposed, this algorithm can select the least cost data sets that are required by the tasks, and moves computation to datanode, completed the join operations in Map stage, reduces the unnecessary data transmission, thus reduces the data access cost greatly, reduces the smart grid processing time, improves the real-time performance. Experimental results show that comparing with SCP algorithm, this algorithm is $27 \%$ lower than it in task completion time, and the selection data has good locality. Verifies the validity of the LCSL algorithm.

At present, due to the limit of laboratory hardware conditions, we can only use the simulation model to verify the validity of the algorithm, but it can already shows advantages that the algorithm has in improving the real-time performance of smart grid task. The next step is to build the real cloud computing platform to test and apply LCSL.

\section{Acknowledgement}

In this paper, the research was sponsored by the National Natural Science Foundation of China ( Project No. 61300040) and Scientific research project of Hebei Province (Project No. Z2012077).

\section{References}

[1] Song Yaqi, Zhou Guoliang, Zhu Yongli, et al . Storage Optimization and Parallel Processing of Power Transmission and Transformation Equipment Condition Monitoring Big Data Based on Cloud Platform[J]. Proceedings of the CSEE, 2015, 35(02): 255-267(in Chinese).

[2] Qu Zhaoyang,Zhu Li,Zhang shilin. Data Processing of Hadoop-based Wide Area measurement System[J]. Automation of Electric Power Systems, 2013, 37(4): 92-97 (in Chinese) .

[3] Cui Jie, Li Taoshen, Lan Hongxing. Design and Development of Mass Data Storage Platform Based on Hadoop[J]. Journal of Computer Research and Development, 2012, 49(z1), 12-18.

[4] Deng Zili. Topology Design and Hadoop Research in cloud computing[D] . University of Science and Technology of China , 2009.

[5] Xia Wei. Research and Improvement of Job Scheduling Algorithm in Hadoop platform [D]. South China University of Technology,2010.

[6] JeffreyDean,SanjayGhemawat. MapReduce: Symplified Data Processing on Large Clusters[J]. NewYork: ACM, 2008, 51(1):107 113.

[7] Jeffrey Dean. Experiences with MapReduce, an abstraction for large-scale computationProc. 15th International Conference on Parallel Architectures and Compilation Techniques,2006:1.

[8] Zhao Yanrong, Wang Weiping, Meng Dan, Zhang Shubin, Li Jun. Efficient Join Query Processing Algorithm CHMJ Based on Hadoop[J]. Journal of Software, 2012,23(8):2032-2041.

[9] Venugopal S,Buyya R.An SCP-based heuristic approach for sche-duling distributed data-intensive application on global grids[J].Journal of Parallel and Distributed Computing,2008,68(4):471-487.

[10] Lu Efeng. Research of Replica Selection Strategies in Data Grid[D]. TianjinUniversity .2006.

[11] Du Wei, Gui Guohua, Liu Wei, Shi Feiyan, Wei Kaizhi. Data Selection Strategy for Data-intensive Applications in Cloud[J]. Computer Science,2012,39(6),30-34+71. 